\title{
Functional cortical reorganization in cases of cervical spondylotic myelopathy and changes associated with surgery
}

\author{
Indira Devi Bhagavatula, MCh, ${ }^{1}$ Dhaval Shukla, MCh, ${ }^{1}$ Nishanth Sadashiva, $M C h,{ }^{1}$ \\ Praveen Saligoudar, $\mathrm{MCh},{ }^{1}$ Chandrajit Prasad, MD, DM, ${ }^{2}$ and Dhananjaya I. Bhat, $\mathbf{M C h}^{1}$
}

Departments of ${ }^{1}$ Neurosurgery and ${ }^{2}$ Neuroimaging and Interventional Radiology, National Institute of Mental Health and Neurosciences, Bangalore, India

OBJECTIVE The physiological mechanisms underlying the recovery of motor function after cervical spondylotic myelopathy (CSM) surgery are poorly understood. Neuronal plasticity allows neurons to compensate for injury and disease and to adjust their activities in response to new situations or changes in their environment. Cortical reorganization as well as improvement in corticospinal conduction happens during motor recovery after stroke and spinal cord injury. In this study the authors aimed to understand the cortical changes that occur due to CSM and following CSM surgery and to correlate these changes with functional recovery by using blood oxygen level-dependent (BOLD) functional MRI (fMRI).

METHODS Twenty-two patients having symptoms related to cervical cord compression due to spondylotic changes along with 12 age- and sex-matched healthy controls were included in this study. Patients underwent cervical spine MRI and BOLD fMRI at 1 month before surgery (baseline) and 6 months after surgery.

RESULTS Five patients were excluded from analysis because of technical problems; thus, 17 patients made up the study cohort. The mean overall modified Japanese Orthopaedic Association score improved in patients following surgery. Mean upper-extremity, lower-extremity, and sensory scores improved significantly. In the preoperative patient group the volume of activation (VOA) was significantly higher than that in controls. The VOA after surgery was reduced as compared with that before surgery, although it remained higher than that in the control group. In the preoperative patient group, activations were noted only in the left precentral gyrus ( $P r C G)$. In the postoperative group, activations were seen in the left postcentral gyrus (PoCG), as well as the PrCG and premotor and supplementary motor cortices. In postoperative group, the VOA was higher in both the PrCG and PoCG as compared with those in the control group.

CONCLUSIONS There is over-recruitment of sensorimotor cortices during nondexterous relative to dexterous movements before surgery. After surgery, there was recruitment of other cortical areas such as the PoCG and premotor and supplementary motor cortices, which correlated with improvement in dexterity, but activation in these areas was greater than that found in controls. The results show that improvement in dexterity and finer movements of the upper limbs is associated with recruitment areas other than the premotor cortex to compensate for the damage in the cervical spinal cord. http://thejns.org/doi/abs/10.3171/2016.3.FOCUS1635

KEY WORDS cervical spondylotic myelopathy; functional magnetic resonance imaging; cortical plasticity

$\mathrm{C}$ ERVICAL spondylotic myelopathy (CSM) is a chronic progressive disease caused by degenerative changes in the spine that results in nerve root and cord compression by osseocartilaginous elements, and spondylosis is the most common cause of neural compression. ${ }^{25}$
Surgery is universally accepted to be better than conservative management with reference to avoiding further neurological deterioration or achieving some functional recovery, ${ }^{2,21}$ although some studies have raised doubts..$^{17,34}$ Various poor prognostic factors for surgery have been de-

ABBREVIATIONS BA = Brodmann area; $B O L D=$ blood oxygen level-dependent; $\mathrm{COG}=$ center of gravity; $\mathrm{CSM}=$ cervical spondylotic myelopathy; FCS = functional connectivity strength; $\mathrm{fMRI}=$ functional MRI; $\mathrm{M} 1$ = primary motor cortex; MEP = motor evoked potential; mJOA = modified Japanese Orthopaedic Association; $\mathrm{N}=$ number of focal points at which MEPs elicited; PMA = premotor area; PoCG = postcentral gyrus; PrCG = precentral gyrus; rsFC = resting-state functional connectivity; $\mathrm{S} 1$ = primary somatosensory cortex; $\mathrm{SCl}=$ spinal cord injury; $\mathrm{SMA}=$ supplementary motor area; $\mathrm{sMEPs}=$ sum amplitude of MEPs; $\mathrm{t}_{\max }=$ maximum $\mathrm{t}$ value; $\mathrm{TMS}=$ transcranial magnetic stimulation; VOA = volume of activation. 
fined in cases of CSM - an older age, abnormal cervical curvature, multisegmental compression, long duration of symptoms, greater number of comorbidities, decreased signal intensity on T1-weighted images, increased signal intensity on T2-weighted images, and cord atrophy on preoperative MR images, to name a few. $6,16,25,30,36,37$ The physiological mechanisms underlying the recovery of motor function after CSM surgery are poorly understood.

Neuronal plasticity allows neurons in the brain and spinal cord to compensate for injury and disease and to adjust their activities in response to new situations or changes in their environment. Two of the most commonly discussed mechanisms by which functional recovery occurs after decompression are synaptic changes and dendritic sprouting in the cortical and spinal cord neurons. ${ }^{12,13}$ Rapid cortical and subcortical changes occurring after spinal cord injury (SCI) or myelitis have been demonstrated on functional imaging. ${ }^{1}$ Cortical reorganization as well as improvement in corticospinal conduction happens during motor recovery after stroke, and this has been proved by studies using transcranial magnetic stimulation (TMS). ${ }^{5,7,31,39}$ Similar functional reorganization of cortical motor functions has been proved in macaque monkeys. ${ }^{35}$ Not many studies have correlated the postoperative motor improvement with plasticity. ${ }^{19}$

Some studies have shown adaptive changes in bilateral primary motor cortex (M1), supplementary motor area (SMA), premotor area (PMA), cingulate motor area, parietal cortex, and contralateral primary somatosensory cortex (S1) in cases of $\mathrm{SCI}^{8-11,27-29,33,40}$ and cervical compressive myelopathy due to spondylosis. ${ }^{13,14,23,38}$ In this study we aimed to understand the cortical changes that occur due to CSM and following CSM surgery and to correlate these changes with functional recovery by using functional MRI (fMRI). We hypothesized that the recovery of normal activation patterns might coincide with clinical improvement.

\section{Methods \\ Patient Selection}

Twenty-two patients having symptoms related to cervical cord compression due to spondylotic changes were selected for the study between 2010 and 2013. Eleven patients had multiple-level disc osteophyte complex with canal stenosis, 8 had single-level prolapsed intervertebral disc, and 6 had multiple-level cervical ossified posterior longitudinal ligament. Inclusion criteria were an age 1870 years, symptoms for at least 2 months- 4 years, weakness of the upper extremities, right handedness, and spine MRI showing 1 or more levels of cervical spinal stenosis and cord compression. Exclusion criteria were previous cervical spine surgery, compromised cognitive function or other neurological or musculoskeletal disease, and an inability to tolerate and meet the safety criteria for repetitive fMRI studies. Twelve age- and sex-matched healthy controls were also recruited for the study. The National Institute of Mental Health and Neurosciences Ethics Committee approved the study. Informed written consent was obtained from all participants or their legal custodians.

\section{Clinical Evaluation and Imaging}

All CSM patients were assessed with the modified Jap- anese Orthopaedic Association (mJOA) scale to record the severity of myelopathy. Patients underwent cervical spine MRI and blood oxygen level-dependent (BOLD) fMRI using a standard paradigm. After a preoperative imaging diagnosis was rendered, patients underwent anterior or posterior decompressive surgery. Postoperatively patients underwent rehabilitation programs and physiotherapy. $\mathrm{Pa}$ tients were monitored up to 6 months after surgery, and the mJOA score was documented. The BOLD fMRI was performed at 6 months using the same motor paradigm. All healthy controls also underwent fMRI with the same paradigm.

\section{Motor Task}

All subjects were instructed to keep their right wrist straight and tap their 4 fingers to their thumb at a constant self-regulated pace. ${ }^{14}$ In all cases, an examiner ensured proper execution of the task during imaging. A block design in which a motor task was alternated with a rest period was used. There were a total of 100 dynamics, and every 10 dynamics of activity was alternated with 10 dynamics of rest, beginning with rest. Healthy volunteers also performed an identical task during fMRI.

\section{Imaging Protocol}

Both patients and healthy controls underwent baseline imaging, and the patients also underwent imaging 6 months after surgery. We performed 3-T MRI (Philips Medical Systems) with a 32-channel head coil. Functional images were acquired using a $\mathrm{T} 2 *$-sensitive spin-echo echo planar imaging sequence sensitive to BOLD contrast. After obtaining the fMR images, we performed 3D highresolution T1-weighted imaging to facilitate the localization of fMRI activation. Anatomical and functional images were processed using Statistical Parametric Mapping 8 (SPM8) software (http://www.fil.ion.ucl.ac.uk/spm/). Preprocessing involved the reorientation of functional and structural images followed by slice timing correction and realignment of functional images.

A hypothesis-driven general linear model (GLM) was used to perform group analyses. Group activation maps were created for each of the 3 groups (control, preoperative, postoperative) using a threshold of $p<0.005$ with Bonferroni correction, which provides stringent protection against false-positives by scaling the $\mathrm{p}$ value by $1 / \mathrm{N}$, where $\mathrm{N}$ is the number of pixels in the functional data set. Based on the results of Curt et al., ${ }^{10}$ a minimum cluster size of $48 \mathrm{~mm}^{3}$ was set to confidently differentiate between likely artifacts and significant regions of activation. For all functional activation maps, the volume of activation (VOA), maximum $\mathrm{t}$ value $\left(\mathrm{t}_{\max }\right)$, and Talairach coordinates of the center of gravity (COG) of the VOA were recorded. Anatomical localization of the functional brain activations was performed using the Talairach Client (www.talairach.org) after converting Montreal Neurological Institute (MNI) coordinates into Talairach coordinates using GingerALE (http://www.brainmap.org/ale/).

\section{Results}

In 4 patients, preoperative fMRI showed poor or no activation. In 1 patient, $\mathrm{x}$-axis movement was more than 5 
mm. Hence, 17 patients with CSM (16 males, mean age $51.76 \pm 10.67$ years) were eligible for analysis in this study. These patients presented with a duration of symptoms ranging from 2 months to 4 years. Twelve patients underwent decompressive laminectomy, and 5 patients underwent an anterior cervical approach and decompression. Spinal implants were not used in any patients. The mean overall mJOA score improved in patients following surgery; mean upper-extremity, lower-extremity, and sensory scores improved significantly (Table 1).

Although our primary intent was to calculate the degree of clinical improvement with the amount of increase/ decrease in VOA, it was not possible given our small sample size. Thus, the mean VOA was calculated in different groups using the software and compared with each other. In the control group, the mean VOA was $529 \mathrm{~mm}^{3}$ in the left $\mathrm{S} 1,190 \mathrm{~mm}^{3}$ in the cerebellum, and $13 \mathrm{~mm}^{3}$ in the right S1. In the patients preoperatively, the VOA was 1001 $\mathrm{mm}^{3}$ in the left S1, $266 \mathrm{~mm}^{3}$ in the cerebellum, and 394 $\mathrm{mm}^{3}$ in the right $\mathrm{S} 1$. In the patients postoperatively, the VOA was $924 \mathrm{~mm}^{3}$ in the left S1, $341 \mathrm{~mm}^{3}$ in the cerebellum, and $39 \mathrm{~mm}^{3}$ in the right S1. The VOA in the contralateral S1 was higher in the patients both preoperatively and postoperatively than that in the controls. The VOA in the cerebellum was higher in the patients both preoperatively and postoperatively than in controls. The VOA in the ipsilateral S1 was higher in the patients preoperatively than in the patients postoperatively and in controls. When sensory and motor cortices were evaluated together for a motor task, the control group showed a VOA of $1072 \mathrm{~mm}^{3}$ (COG-Brodmann area [BA] 4). In the preoperative patient group, the VOA was significantly higher $\left(4305 \mathrm{~mm}^{3}\right.$, COG-BA4) than that in controls. Following surgery, the VOA was reduced (2945 $\mathrm{mm}^{3}$, COG-BA3) as compared with that preoperatively, but it remained higher than that in the control group (Fig. 1 and Table 2).

Further analysis of the sensorimotor cortex demonstrated the different responses to surgical intervention between the sensory and motor cortices (Table 3 ). In the preoperative group, activations increased and were noted only in the left precentral gyrus (PrCG; COG-BA4, VOA 5998 $\mathrm{mm}^{3}$ ). In the postoperative group, activations were seen in the left postcentral gyrus (PoCG; COG-BA3, VOA 736 $\mathrm{mm}^{3}$ ), as well as the PrCG and the premotor and supplementary motor cortices (COG-BA6, VOA $736 \mathrm{~mm}^{3}$ ). The PoCG VOA was significantly higher in the postoperative group $\left(654 \mathrm{~mm}^{3}\right)$ than in the control group. In the postoperative group, activations were seen in the PMA and SMA $\left(736 \mathrm{~cm}^{3}\right)$, which were not seen in controls or the

TABLE 1. Preoperative and postoperative mean mJOA scores*

\begin{tabular}{lcc}
\hline \multicolumn{1}{c}{ mJOA Score } & Preop & Postop \\
\hline Upper-extremity motor score $\dagger$ & $2.65 \pm 1.05$ & $4.24 \pm 0.97$ \\
\hline Lower-extremity motor score $\dagger$ & $3.65 \pm 0.93$ & $5.47 \pm 1.17$ \\
\hline Sensory score $\dagger$ & $1.82 \pm 0.39$ & $2.41 \pm 0.5$ \\
\hline Sphincter score & $2.41 \pm 0.61$ & $2.71 \pm 0.47$ \\
\hline Total† & $10.71 \pm 2.64$ & $14.88 \pm 2.49$ \\
\hline
\end{tabular}

* Values expressed as the means \pm standard deviation.

$\dagger p<0.05$, paired Student t-test. preoperative group. In the postoperative group, the VOA was higher in both the PrCG and PoCG as compared with those in the control group.

\section{Discussion}

Neuronal plasticity leads to the reorganization of cortical networks in the brain after neurological injury. Various studies have tried to explain and show how reorganization occurs in the cortical and subcortical levels in cases of disease and temporary dysfunction by using advanced neuroimaging and brain mapping along with standard electrophysiological testing. The reorganization can be both structural and functional. Structural changes include synaptic alterations like a change in the length and diameter of existing dendritic branches or the growth of new branches, providing the opportunity for new synapses. ${ }^{3}$ Functional changes can be modifications in neuronal activity or synaptic efficacy ${ }^{15}$ or increases in astrocytic activity. ${ }^{4}$ Presently many modalities, such as fMRI, PET, electroencephalography, TMS, and magnetic encephalography, permit monitoring of brain activation while movement is taking place.

There is evidence that SCI results in the reorganization of motor areas in the brain. Increased activation in secondary brain areas as well as a distinct spatial shift in activation compared with that in controls has been demonstrated. ${ }^{29}$ Patients with complete SCI exhibit morphological changes such as reduced gray matter volume in the primary motor, medial prefrontal, cingulate, and cerebellar cortices, as well as diffusion tensor imaging changes in cortical and brainstem motor areas..$^{14,22,41}$ With the advent of functional imaging, various reorganization patterns were recognized. In a review by Kokotilo et al. ${ }^{29}$ nearly half of the included studies documented a significant increase in activation magnitude in motor areas. Some studies found a distinct spatial shift in activation, with a majority showing a posterior shift in activation and 4 studies demonstrating a shift in activation toward deafferented limb representation. ${ }^{29}$ Compared with SCI in which spontaneous recovery happens depending on a variety of factors such as time since injury, lesion level, completeness of injury, and amount of deafferentation, CSM provides a unique model of SCI with potentially reversible neurological changes when treated with surgery.

In some early studies, postoperative cord expansion was proposed as a good indicator of recovery and was related to "intracord plasticity," though the researchers did not use electrophysiology or functional brain imaging. ${ }^{1,18}$ In one of the earliest studies in which functional imaging was used to assess cortical reorganization in CSM, Holly et al. ${ }^{23}$ demonstrated expansion of the cortical representation of the affected extremity (involvement of adjacent motor territories, the SMA, and bilateral cortical recruitment) before surgery in 4 patients, and surgical decompression resulted in improvements in neurological function and reorganization of the representational map.

In a larger study by Dong et al., ${ }^{13}$ which included 8 patients with CSM and 6 healthy control volunteers, the authors quantitatively captured reduced activation in the contralateral M1, S1, and dorsal PMA for dexterous fin- 

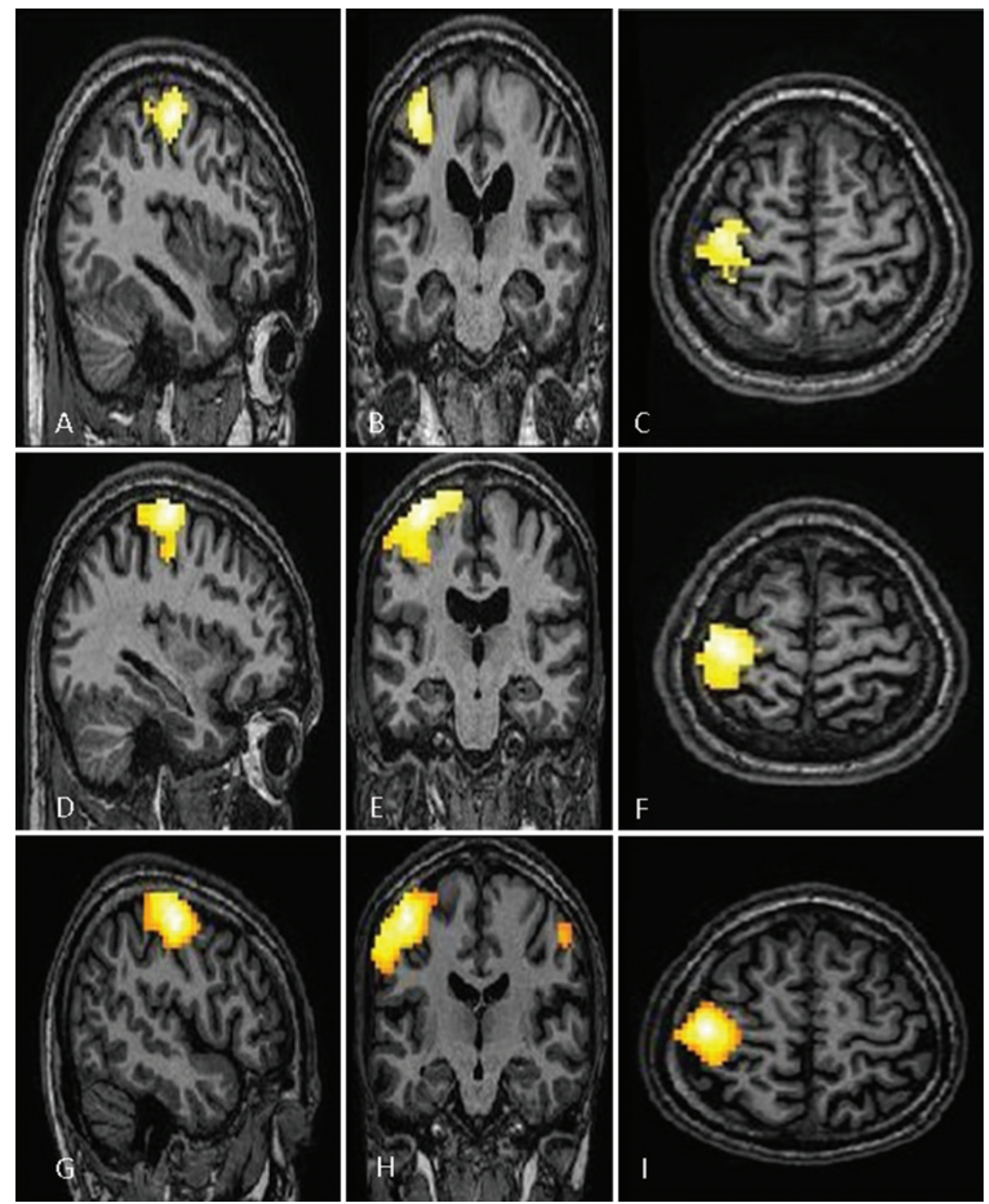

FIG. 1. Control (A, B, and C), preoperative (D, E, and F), and postoperative (G, H, and I) images (extent threshold $\mathrm{k}=48 \mathrm{~mm}^{3}, \mathrm{p}$ $<0.005$, Bonferroni corrected) showing activity in the left sensory and motor cortices following right finger tapping. Functional images have been superimposed on the structural images for better visualization. Yellow areas signify strong activation, and orange areas signify weak activation.

ger movements-though not for less complex wrist movements before surgery-followed by a progressive increase toward the activation pattern of healthy controls, as functional gains in the upper extremity proceeded after surgical decompression. The reduced pinch-related activation and the normal amount of wrist extension-related activation in contralateral M1 and S1 before surgery suggest that the coordinated finger pinch task demands greater integrity of the corticospinal tract. They postulated that contralateral hemisphere changes during dexterous pinch movements might suggest that some nonpermanently damaged axons within descending motor and ascending sensory pathways have the potential for reversibility of a conduction block. Thus, more cortical sensorimotor neurons were recruited postsurgery as a result of enhanced corticospinal conductivity, and by halting the progression of corticospinal tract injury, cortical sensorimotor reorganization evolved to compensate for lost axons.

In a study by Duggal et al., ${ }^{14}$ fMRI was performed in 12 patients prior to decompression and 6 months following surgery. These authors demonstrated that spinal cord compression results in an increased VOA within the PrCG and a loss in VOA within the PoCG in comparison with those in controls. Following decompression, there were varying degrees of increased activation in both the PrCG and PoCG, suggesting cortical recruitment and regional reorganization.

In their study involving 7 patients, Hrabálek et al. ${ }^{24}$ 
TABLE 2. Total VOA in the left sensorimotor cortices in different groups

\begin{tabular}{lcccc}
\hline Group & VOA $\left(\mathrm{mm}^{3}\right)$ & $\mathrm{t}_{\max }$ & Talairach Coordinates $(\mathrm{x}, \mathrm{y}, \mathrm{z})$ & $\mathrm{COG}$ \\
\hline Control & 1072 & 14.03 & $-30.41,-28.77,50.58$ & BA4 \\
\hline Preop & 4305 & 11.59 & $-33.18,-25.96,50.8$ & BA4 \\
\hline Postop & 2945 & 15.64 & $-35.97,-28.74,50.49$ & BA3 \\
\hline
\end{tabular}

performed preoperative and 6-month postoperative fMRI studies with the task paradigm of wrist flexion and extension. Postoperatively, the anteroposterior cervical spinal canal distance increased by $40 \%$, the cross-sectional area of the spinal canal by $37 \%$, and cross-sectional areas of the spinal cord by $36 \%$. Functional MRI revealed significant activation in the dorsal M1 and adjacent secondary motor and sensory areas, bilaterally in the SMAs, anterior cingulum, primary auditory cortex basal ganglia, thalamus, and cerebellum. After surgery, the cortical activations decreased in most areas. There was a statistically significant decrease in activation of the right parietal operculum and posterior temporal lobe.

In a case report by Tam et al., ${ }^{38}$ although no preoperative fMRI studies were available, postdecompression fMRI detected increased cortical activation in the M1 during finger tapping with concomitant improvement in motor function. Significant activation was also seen in the ipsilateral culmen of the cerebellum, contralateral medial frontal gyrus, and contralateral inferior parietal lobule. Postoperatively, the patient demonstrated 2 VOAs in the contralateral PrCG and $1 \mathrm{VOA}$ in the ipsilateral PrCG without any activation in the PoCG.

In a recent study, Zhou et al. ${ }^{42}$ studied functional connectivity strength (FCS) via resting-state functional connectivity (rsFC) MRI of the sensorimotor network. Patients with CSM showed significantly decreased FCS in the operculum-integrated regions, which exhibited reduced rsFC around the rolandic sulcus. They also showed increased FCS in the premotor, primary somatosensory, and parietal-integrated areas, which primarily showed an enhanced rsFC pattern. Altered FCS was associated with worsening JOA scores.

Green et al. ${ }^{19}$ performed TMS and motor function testing 1 month prior to and 4 months after surgery for CSM. They showed that the sum amplitude of motor evoked potentials (sMEPs) and number of focal points at which MEPs were elicited (N) were significantly greater in CSM patients than in controls. Patients with mixed upper and lower limb dysfunction had significantly increased grip strength and reduced sMEPs and $\mathrm{N}$ after surgery. Patients with only lower limb dysfunction did not show significant reduction in sMEPs or $\mathrm{N}$ after surgery. No significant differences in Hoffmann's reflex parameters obtained from the flexor carpi radialis muscle or in central motor conduction time (CMCT) changes were noted after surgery. These authors proved that the compensatory expansion of motor cortical representation occurs largely at the cortical rather than the spinal levels, with a tendency to normalization after surgery.

In our study there was an increase in VOA in the PrCG
TABLE 3. Volume of activation in different groups in different areas

\begin{tabular}{cccccc}
\hline \multirow{2}{*}{ Group } & BA & Gyrus & $\begin{array}{c}\text { VOA } \\
\left(\mathrm{mm}^{3}\right)\end{array}$ & $\mathrm{t}_{\max }$ & COG $(\mathrm{x}, \mathrm{y}, \mathrm{z})$ \\
\hline Control & 4 & Lt PrCG & 418 & 14.03 & $-30.41,-28.77,50.58$ \\
\cline { 2 - 6 } & 2 & Lt PoCG & 654 & 11.62 & $-49.73,-25.09,-42.5$ \\
\hline Preop & 4 & Lt PrCG & 5998 & 12.606 & $-36.02,-20.88,-56.64$ \\
\cline { 2 - 6 } & 3 & Lt PoCG & - & - & - \\
\hline Postop & 4 & Lt PrCG & 1473 & 15.64 & $-35.97,-28.74,50.49$ \\
\cline { 2 - 6 } & 2 & Lt PoCG & 736 & 7.61 & $-2.6,-9.35,52.89$ \\
\cline { 2 - 6 } & 6 & Lt PMA \& & 736 & 7.62 & $-46.82,-26.85,31.57$ \\
& \multicolumn{5}{c}{ SMA } \\
\hline
\end{tabular}

compared with that in controls. This finding can be explained as the over-recruitment of sensorimotor cortices during nondexterous relative to dexterous movements occurring before surgery. After surgery, other cortical areas such as the PoCG and the premotor and supplementary motor cortices were recruited. The SMA is involved in the preparation and execution of practiced movement sequences and is strongly influenced by attention, performance, and other components of the movement, ${ }^{20}$ which had actually improved after surgery in our cases, as reflected by improved upper limb mJOA scores. Dorsal premotor cortex is proved to be involved in the recovery of dexterity after stroke and SCI, possibly through corticospinal and corticorubral projections to spinal motor neurons in the midcervical segments. ${ }^{26,32}$ Regarding the posterior shift of activation toward the PoCG, previous studies have already documented the posterior spread of activation with the recovery of limb motor function or increased VOA following injury. ${ }^{10,23}$

Given the existing literature, we can accept that there is compensatory expansion and increased cortical representation of the hand area preoperatively. However, postoperatively, the tendency to normalization of motor representation is less well understood and inconsistent. Patients with CSM form a heterogeneous group with different levels of impairment with different degrees of cord compromise and may have different natural histories if they go untreated. Additionally the extent of synaptic transmission and reorganization depends on time after the initial insult and variable degree of residual spinal cord atrophy. There is no uniformity in the timing of imaging in relation to surgery, and different authors have used different tasks for fMRI, which makes it additionally difficult to come to any conclusion. All reported studies have identified altered cortical sensorimotor activation patterns together but have not attempted to make distinctions between the motor and sensory responses separately. Somatosensory cortical atrophy can occur in patients with chronic disease, so the VOA may provide incorrect values in comparison with those in healthy controls as the basal brain volume levels are not matched.

Another explanation for the increase in S1 activity during dexterous movements and behavioral improvement postoperatively may be that corticospinal axons originat- 
ing from S1 are more posterior and medial than those of M1 and less vulnerable to compression. Reorganization after injury and surgery may be correlated to a patient's functional recovery, but there may be multiple confounding factors, such as rehabilitative physiotherapy, that produce more sensory activation and thereby increase coactivation of the sensory cortex. A more conclusive study must incorporate a larger number of patients with similar clinical features and duration of symptoms, demonstrate the central motor conduction time, and correlate TMS studies with areas of fMRI activation before surgery and periodically in the postoperative period. Resting-state fMRI may be an effective platform to explore neuronal functional connectivity and may help in further understanding plasticity without any confounding factors associated with task-related imaging.

\section{Conclusions}

There is an increase in VOA during movements in cases of CSM to compensate for weakness and loss of dexterity compared with controls. Postoperatively, though the compression is relieved, there is cortical reorganization with the recruitment of PMA and SMAs in an attempt to gain dexterity and coordination. The reorganization signifies dynamic cortical plasticity in response to surgery.

\section{References}

1. Baba H, Maezawa Y, Uchida K, Furusawa N, Wada M, Imura S: Plasticity of the spinal cord contributes to neurological improvement after treatment by cervical decompression. A magnetic resonance imaging study. J Neurol 244:455-460, 1997

2. Baskin DS, Ryan P, Sonntag V, Westmark R, Widmayer MA: A prospective, randomized, controlled cervical fusion study using recombinant human bone morphogenetic protein-2 with the CORNERSTONE-SR allograft ring and the ATLANTIS anterior cervical plate. Spine (Phila Pa 1976) 28:1219-1225, 2003

3. Bayona NA, Bitensky J, Teasell R: Plasticity and reorganization of the uninjured brain. Top Stroke Rehabil 12:1-10, 2005

4. Benloucif S, Bennett EL, Rosenzweig MR: Norepinephrine and neural plasticity: the effects of xylamine on experienceinduced changes in brain weight, memory, and behavior. Neurobiol Learn Mem 63:33-42, 1995

5. Byrnes ML, Thickbroom GW, Phillips BA, Mastaglia FL: Long-term changes in motor cortical organisation after recovery from subcortical stroke. Brain Res 889:278-287, 2001

6. Chatley A, Kumar R, Jain VK, Behari S, Sahu RN: Effect of spinal cord signal intensity changes on clinical outcome after surgery for cervical spondylotic myelopathy. J Neurosurg Spine 11:562-567, 2009

7. Cicinelli P, Traversa R, Rossini PM: Post-stroke reorganization of brain motor output to the hand: a 2-4 month follow-up with focal magnetic transcranial stimulation. Electroencephalogr Clin Neurophysiol 105:438-450, 1997

8. Cramer SC, Lastra L, Lacourse MG, Cohen MJ: Brain motor system function after chronic, complete spinal cord injury. Brain 128:2941-2950, 2005

9. Crawley AP, Jurkiewicz MT, Yim A, Heyn S, Verrier MC, Fehlings MG, et al: Absence of localized grey matter volume changes in the motor cortex following spinal cord injury. Brain Res 1028:19-25, 2004
10. Curt A, Alkadhi H, Crelier GR, Boendermaker SH, HeppReymond MC, Kollias SS: Changes of non-affected upper limb cortical representation in paraplegic patients as assessed by fMRI. Brain 125:2567-2578, 2002

11. Curt A, Bruehlmeier M, Leenders KL, Roelcke U, Dietz $\mathrm{V}$ : Differential effect of spinal cord injury and functional impairment on human brain activation. J Neurotrauma 19:43-51, 2002

12. Dobkin BH: Confounders in rehabilitation trials of taskoriented training: lessons from the designs of the EXCITE and SCILT multicenter trials. Neurorehabil Neural Repair 21:3-13, 2007

13. Dong Y, Holly LT, Albistegui-Dubois R, Yan X, Marehbian J, Newton JM, et al: Compensatory cerebral adaptations before and evolving changes after surgical decompression in cervical spondylotic myelopathy. J Neurosurg Spine 9:538-551, 2008

14. Duggal N, Rabin D, Bartha R, Barry RL, Gati JS, Kowalczyk $\mathrm{I}$, et al: Brain reorganization in patients with spinal cord compression evaluated using fMRI. Neurology 74:1048-1054, 2010

15. Dunlop SA: Activity-dependent plasticity: implications for recovery after spinal cord injury. Trends Neurosci 31:410 418, 2008

16. Fehlings MG, Arvin B: Surgical management of cervical degenerative disease: the evidence related to indications, impact, and outcome. J Neurosurg Spine 11:97-100, 2009

17. Fouyas IP, Statham PF, Sandercock PA, Lynch C: Surgery for cervical radiculomyelopathy. Cochrane Database Syst Rev (3):CD001466, 2001

18. Fukushima T, Ikata T, Taoka Y, Takata S: Magnetic resonance imaging study on spinal cord plasticity in patients with cervical compression myelopathy. Spine (Phila Pa 1976) 16 (10 Suppl):S534-S538, 1991

19. Green A, Cheong PWT, Fook-Chong S, Tiruchelvarayan R, Guo CM, Yue WM, et al: Cortical reorganization is associated with surgical decompression of cervical spondylotic myelopathy. Neural Plast 2015:389531, 2015

20. He SQ, Dum RP, Strick PL: Topographic organization of corticospinal projections from the frontal lobe: motor areas on the medial surface of the hemisphere. J Neurosci 15:32843306, 1995

21. Henderson FC, Geddes JF, Vaccaro AR, Woodard E, Berry KJ, Benzel EC: Stretch-associated injury in cervical spondylotic myelopathy: new concept and review. Neurosurgery 56:1101-1113, 2005

22. Henderson LA, Gustin SM, Macey PM, Wrigley PJ, Siddall PJ: Functional reorganization of the brain in humans following spinal cord injury: evidence for underlying changes in cortical anatomy. J Neurosci 31:2630-2637, 2011

23. Holly LT, Dong Y, Albistegui-DuBois R, Marehbian J, Dobkin B: Cortical reorganization in patients with cervical spondylotic myelopathy. J Neurosurg Spine 6:544-551, 2007

24. Hrabálek L, Hluštík P, Hok P, Wanek T, Otruba P, Cecháková $\mathrm{E}$, et al: [Effects of spinal cord decompression in patients with cervical spondylotic myelopathy oncortical brain activations.] Rozhl Chir 93:530-535, 2014 (Czech)

25. Ishii M, Wada E, Hamada M: Deterioration of surgical outcomes with aging in patients with cervical spondylotic myelopathy. J Spinal Disord Tech 25:E199-E203, 2012

26. Johansen-Berg H, Dawes H, Guy C, Smith SM, Wade DT, Matthews PM: Correlation between motor improvements and altered fMRI activity after rehabilitative therapy. Brain 125:2731-2742, 2002

27. Jurkiewicz MT, Crawley AP, Verrier MC, Fehlings MG, Mikulis DJ: Somatosensory cortical atrophy after spinal cord injury: a voxel-based morphometry study. Neurology 66:762-764, 2006

28. Jurkiewicz MT, Mikulis DJ, McIlroy WE, Fehlings MG, Ver- 
rier MC: Sensorimotor cortical plasticity during recovery following spinal cord injury: a longitudinal fMRI study. Neurorehabil Neural Repair 21:527-538, 2007

29. Kokotilo KJ, Eng JJ, Curt A: Reorganization and preservation of motor control of the brain in spinal cord injury: a systematic review. J Neurotrauma 26:2113-2126, 2009

30. Lemcke J, Menz H, Al-Zain F, Meier U: Outcome after anterior cervical discectomy and arthrodesis: a clinical study of 368 patients. Neurosurg Q 20:8-14, 2010

31. Malcolm MP, Triggs WJ, Light KE, Shechtman O, Khandekar G, Gonzalez Rothi LJ: Reliability of motor cortex transcranial magnetic stimulation in four muscle representations. Clin Neurophysiol 117:1037-1046, 2006

32. Mazevet D, Meunier S, Pradat-Diehl P, Marchand-Pauvert $\mathrm{V}$, Pierrot-Deseilligny E: Changes in propriospinally mediated excitation of upper limb motoneurons in stroke patients. Brain 126:988-1000, 2003

33. Mikulis DJ, Jurkiewicz MT, McIlroy WE, Staines WR, Rickards L, Kalsi-Ryan S, et al: Adaptation in the motor cortex following cervical spinal cord injury. Neurology 58:794801,2002

34. Nikolaidis I, Fouyas IP, Sandercock PA, Statham PF: Surgery for cervical radiculopathy or myelopathy. Cochrane Database Syst Rev (1):CD001466, 2010

35. Nishimura Y, Onoe H, Morichika Y, Perfiliev S, Tsukada H, Isa T: Time-dependent central compensatory mechanisms of finger dexterity after spinal cord injury. Science 318:11501155,2007

36. Rao RD, Gourab K, David KS: Operative treatment of cervical spondylotic myelopathy. J Bone Joint Surg Am 88:1619_ 1640, 2006

37. Sampath P, Bendebba M, Davis JD, Ducker TB: Outcome of patients treated for cervical myelopathy. A prospective, multicenter study with independent clinical review. Spine (Phila Pa 1976) 25:670-676, 2000

38. Tam S, Barry RL, Bartha R, Duggal N: Changes in functional magnetic resonance imaging cortical activation after decompression of cervical spondylosis: case report. Neurosurgery 67:E863-E864, 2010

39. Thickbroom GW, Byrnes ML, Archer SA, Mastaglia FL:
Motor outcome after subcortical stroke correlates with the degree of cortical reorganization. Clin Neurophysiol 115:2144-2150, 2004

40. Topka H, Cohen LG, Cole RA, Hallett M: Reorganization of corticospinal pathways following spinal cord injury. Neurology 41:1276-1283, 1991

41. Wrigley PJ, Gustin SM, Macey PM, Nash PG, Gandevia SC, Macefield VG, et al: Anatomical changes in human motor cortex and motor pathways following complete thoracic spinal cord injury. Cereb Cortex 19:224-232, 2009

42. Zhou FQ, Tan YM, Wu L, Zhuang Y, He LC, Gong HH: Intrinsic functional plasticity of the sensory-motor network in patients with cervical spondylotic myelopathy. Sci Rep 5:9975, 2015

\section{Disclosures}

The authors report no conflict of interest concerning the materials or methods used in this study or the findings specified in this paper.

\section{Author Contributions}

Conception and design: Bhagavatula, Shukla, Saligoudar. Acquisition of data: Shukla, Sadashiva, Saligoudar, Prasad. Analysis and interpretation of data: Sadashiva, Saligoudar, Prasad. Drafting the article: Bhagavatula, Sadashiva, Saligoudar, Bhat. Critically revising the article: Bhagavatula, Shukla, Sadashiva, Bhat. Reviewed submitted version of manuscript: Bhagavatula, Sadashiva. Approved the final version of the manuscript on behalf of all authors: Bhagavatula. Statistical analysis: Sadashiva, Prasad. Administrative/technical/material support: Bhagavatula, Shukla, Saligoudar, Prasad, Bhat. Study supervision: Bhagavatula, Shukla, Saligoudar, Prasad, Bhat.

\section{Correspondence}

Indira Devi Bhagavatula, Department of Neurosurgery, 2nd Fl., Neurosciences Faculty Building, National Institute of Mental Health and Neurosciences, Hosur Rd., Bangalore 560029, India. email: bidevidr@gmail.com. 